\title{
Directing the Growth of Highly-Aligned Gold Nanorods through a Surface Chemical Amidation Reaction
}

\author{
Aneta J. Mieszawska, Grzegorz W. Slawinski, and Francis P. Zamborini
}

\section{Supporting Information}

Detailed procedure for growth of aligned $\mathrm{Au}$ and Ag NRs on the surface:

Aligned Au NRs were synthesized directly on Si (100) substrates as shown in Scheme 1 of the paper. First, a Si (100) substrate with a native oxide layer was functionalized with aminopropyltriethoxysilane (APTES) by cleaning in $3: 1 \mathrm{H}_{2} \mathrm{SO}_{4}: \mathrm{H}_{2} \mathrm{O}_{2}$ (piranha) solution (Caution: piranha reacts violently with organic compounds), rinsing thoroughly with water and isopropanol (IPA), and placing into a solution containing $100 \mu \mathrm{L}$ APTES, $10 \mathrm{~mL}$ IPA, and 2-3 drops of water while heating just below boiling for $30 \mathrm{~min}$. The substrate was removed, rinsed thoroughly with IPA, dried under $\mathrm{N}_{2}$, and placed into a $10 \mathrm{~mL}$ methylene chloride solution containing $10 \mathrm{mM}$ acetic acid, $20 \mathrm{mM}$ dicyclohexylcarbodiimide (DCC), and $20 \mathrm{mM}$ dimethylamino pyridine (DMAP) for $4 \mathrm{~h}$. The substrate was removed, rinsed with methylene chloride and IPA, and dried under $\mathrm{N}_{2}$. This procedure resulted in the formation of an amide bond between the surface $\mathrm{NH}_{2}$ groups and the $\mathrm{COOH}$ group from acetic acid. 3-5 nm average diameter citrate-capped $\mathrm{Au}$ nanoparticles (NPs) were prepared as previously described ${ }^{1}$ and attached to unreacted amine groups through electrostatic or $\mathrm{Au}-\mathrm{NH}_{2}$ interactions by simply immersing the substrate into the Au NP solution $\left(2.5 \times 10^{-4} \mathrm{M}\right.$ in $\left.\mathrm{Au}\right)$ for $15 \mathrm{~min}$. The substrate was rinsed with water, dried under $\mathrm{N}_{2}$, and placed into a "growth solution" containing $9 \mathrm{~mL} 0.1 \mathrm{M}$ cetyltrimethylammonium bromide (CTAB), $450 \mu \mathrm{L} \quad 0.01 \mathrm{M}$ $\mathrm{HAuCl}_{4}$, and $50 \mu \mathrm{L} 0.1 \mathrm{M}$ ascorbic acid for $1 \mathrm{~h}$. This step leads to the growth of the Au NP seeds into Au NRs via seed-mediated growth from citrate-stabilized $\mathrm{Au}$ nanoparticles in solution ${ }^{1 \mathrm{f}}$ or attached to surfaces. ${ }^{1 \mathrm{lac}}$ The same method was used to prepare Ag NRs, except that the substrate was immersed into 10.60 buffered Ag growth solution, containing $10 \mathrm{~mL}$ of $0.1 \mathrm{M} \mathrm{CTAB}, 250 \mu \mathrm{L} 0.01 \mathrm{M} \mathrm{AgNO}_{3}$, and $500 \mu \mathrm{L}$ $0.1 \mathrm{M}$ ascorbic acid, for $30 \mathrm{~min}$ instead of Au growth solution.

\section{Visible Spectra of Samples Prepared on Glass Slides:}

Figure S1, frame A shows the visible spectra of $\mathrm{Au}$ NPs attached to glass/APTES and glass/APTES/acetic acid (spectrum 1 and 3, respectively). Spectrum 1 shows a clear surface plasmon (SP) band at $\sim 525 \mathrm{~nm}$ consistent with a high density of $\mathrm{Au}$ NPs attached to the surface $\mathrm{NH}_{2}$ groups. The low absorbance and absence of a SP band for Au NPs attached to glass/APTES/acetic acid (spectrum 3) shows that the amidation reaction was successful and blocked the accessibility of most of the $\mathrm{NH}_{2}$ groups. Spectrum 2 and 4 show the two surfaces after exposure to growth solution, respectively. The absorbance of the glass/APTES slide decreases slightly after growth, which is thought to be due to the removal of particles from the surface by the highly-concentrated surfactant, CTAB. ${ }^{\mathrm{Ib}}$ The absorbance of the glass/APTES/acetic acid surface after growth solution in contrast increases slightly and exhibits a noticeable SP band due to the growth of the Au NP seeds into aligned $\mathrm{Au}$ NRs and larger particles. These spectra are consistent with

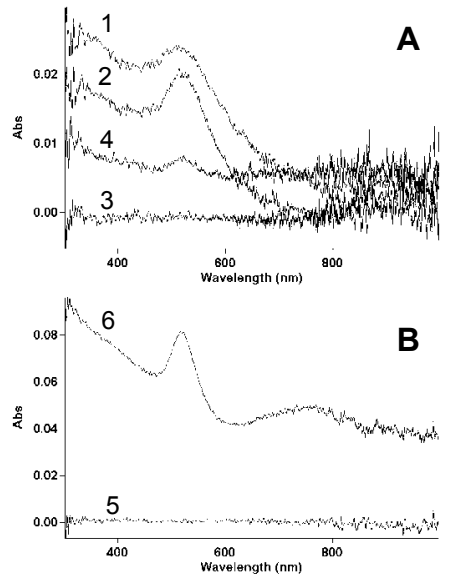

Figure S1. UV-vis spectra of (A) glass/APTES/Au NP before (1) and after (2) growth solution and glass/APTES/acetic acid/Au NP before (3) and after (4) growth solution; (B) glass/MPTMS/Au NP before (5) and after (6) growth solution.

the coverages observed in the Figure 1 SEM images of the main paper.

Figure B, spectrum 5 and 6, shows a glass/MPTMS surface placed in Au NP seed and then growth solution, respectively, for comparison. After seed, spectrum 5 shows no evidence of the Au SP band due to 
the low coverage of Au seed NPs on the surface. After growth (spectrum 6), the absorbance increases dramatically due to the growth of seeds into larger particles and NRs. Consistent with the coverages observed by SEM (Figure 1, main paper), the absorbance is larger compared to glass/APTES/acetic acid (spectrum 4).

Reference 1 is cited in the main paper. 